International Journal of Current Micro6iology and Applied Sciences

ISSN: 2319-7706 Volume 6 Number 2 (2017) pp. 1567-1573

Journal homepage: http://www.ijcmas.com

Original Research Article

http://dx.doi.org/10.20546/ijcmas.2017.602.175

\title{
Detection of Metallo Beta Lactamase Producing Pseudomonas aeruginosa among Clinical Isolates
}

\author{
R. Sujatha*, Roma Goyal and Vikas Mishra \\ Department of Microbiology, Rama Medical College, Kanpur, India \\ *Corresponding author:
}

\section{A B S T R A C T}

\section{Keywords \\ Metallo beta \\ lactamase(MBL), \\ Pseudomonas \\ aeruginosa, E-test, \\ Modified Hodge test, Imepenem.}

Article Info

Accepted:

24 January 2017

Available Online:

10 February 2017
Pseudomonas aeruginosa is an opportunistic pathogenic bacterium responsible for both acute and chronic infection causing serious infections in patients who are mechanically ventilated individuals, who are immunocompromised, and patients with malignancies or HIV infection. The present study was undertaken with the aim to find prevalence of Metallo- $\beta$-Lactamases producing Pseudomonas aeruginosa among clinical isolates by different phenotypic confirmatory methods. The study was carried out in the Department of Microbiology, Rama Medical College, Kanpur for the period between January 2016 to August 2016. A Total of 86 Pseudomonas aeruginosa isolated from different clinical samples including urine, sputum, ear swab, wound swab specimens, pus were identified by standard microbiological techniques \& the isolates were further tested for MBL by screening test, and all screening positive were confirmed by Imipenem - EDTA combined disc test, Modified Hodge test and MBL E test (Imipenem). Out of 86 Pseudomonas aeruginosa isolated $29(33.7 \%$ ) were screening test-positives by imipenem, meropenam and third generation cephalosporins. Of which $14(48.2 \%)$ were MBL positive by Imipenem - EDTA combined disc test, 15(51.7\%) by Modified Hodge test and 19(65.5\%) by MBL E test. MBL E-Test followed by modified Hodge test was found to be the best phenotypic method for detection of MBL. The appearance of the Metallo- $\beta$-Lactamases and their spread among bacterial pathogens is a matter of concern with regard to the future of antimicrobial therapy. So, MBL detection method is recommended into the daily routine clinical laboratory.

\section{Introduction}

Pseudomonas aeruginosa is an increasing prevalent opportunistic human pathogen most common gram-negative bacteria found in nosocomial infections. Despite improvements in antibiotic therapy, Pseudomonas aeruginosa is intrinsically resistant to a number of antimicrobial agents. ${ }^{[1]}$ It is mainly present as a saprophyte in warm moist conditions in the environment including sinks, drains, respirators, humidifiers and

\section{disinfectant solutions.}

The chances of Pseudomonas aeruginosa, carriage increases in the nosocomial infection, reaching 30\% after 3 weeks, Pseudomonas aeruginosa that is encountered in healthy individuals has emerged as the aetiological agent in hospitalized patients (Nosocomial infection) with impaired immune defenses. ${ }^{[2]}$ Pseudomonas aeruginosa causes urinary tract 
infections(UTI), bloodstream infections, intraabdominal infections, and ventilator associated pneumonia(VAP).

Among the beta lactams, carbapenems are considered as the potent drug for serious treatment of gram-negative bacteria infections. ${ }^{[3]}$ Worldwide, the prevalence of gram negative bacteria with multi-drug resistance profiles is now recognized. ${ }^{[4,5,6]}$ However, over the decades resistance to carbapenems is caused due to production of carbepenemases have been reported. Carbapenemases acquires resistance belongs to Ambler molecular classes A, B and D. Metallo-beta-lactamases (MBL) enzymes are the most significant carbapenemases. ${ }^{[7,8,9]}$

It belongs to class $\mathrm{B}$ require divalent cations as cofactors for optimum enzyme activity, and are inhibited by the action of metal ion chelator. They hydrolyse all the beta lactams including carbapenems except the monobactams such as aztreonem. these strains shows resistance towards different classes of antimicrobial agents shows transferable properties towards various types of bacteria. $^{[8,10]}$ Infections caused by MBL producing organisms are associated with high rate of mortality, morbidity and rising healthcare costs because of less alternative of treatment left.

In early 1990s an integron mediated metallo $\beta$-lactamase gene was first isolated among enterobacteriacae, Pseudomonas and other non fastidious gram negative bacilli isolated in several hospitals in Japan. ${ }^{[1]]}$ Over the last decade MBL producing isolates have emerged in Pseudomonas aeruginosa mainly. These isolates are responsible for serious infections such as septicaemia and pneumonia and responsible for failure of therapy with carbapenems. In recent years, resistance to carbapenem among members of Enterobacteriaceae and non fermenters have become a major health related concern worldwide. $^{[12]}$

Clinical infection with such organisms is responsible for serious therapeutic challenges with increasing reports of poor patients outcomes and morbidity.

The aim of this study is to find the prevalence of MBL production in Pseudomonas aeruginosa among different clinical isolates. To evaluate different screening methods for detection of Metallo- $\beta$ lactamase (MBL) production in Pseudomonas aeruginosa and to find out the minimum inhibition concentration(MIC) by E-test.

\section{Materials and Methods}

This study was a prospective study including laboratory investigations and observational analytical design. The study was conducted in Rama Medical College Hospital \& Research centre, Kanpur, U.P. A total of 86 Pseudomonas aeruginosa isolated from different clinical samples including urine, sputum, ear swab, wound swab, pus sent to the microbiology laboratory for the culture identification and sensitivity testing were included in the study.

MBL Screening methods: Antimicrobial susceptibility of Pseudomonas aeruginosa was performed by the disc- diffusion method (Modified- Kirby baur disc diffusion method) as per CLSI guidelines. ${ }^{[13]}$ Isolates resistant to imipenem, meropenem, ertapenem, and third generation cephalosporin were considered as screening positive. Quality control strains that were used are Escherichia coli- American type culture collection(ATCC) 25922 and Pseudomonas aeruginosa - ATCC 27853 .

\section{Phenotypic confirmatory test}

Modified Hodge Test: The modified Hodge 
Test (MHT) detects carbepenemase production in gram negative isolates. An overnight culture suspension of Escherichia coli ATCC 25922 adjusted to 0.5 McFarland was inoculated using a sterile cotton swab on the surface of Muller-Hinton agar(MHA).

After drying, $10 \mu \mathrm{g}$ imipenem disk(Hi-Media, Mumbai, India) was kept at the centre of the MHA plate and the test strains suspespension was inoculated by streaking method from the edge of the imipenem disc to the periphery of the petriplate in four different directions. The plates was incubated overnight at optimum temperature. If the test strain that are carbenemase producing there will be the presence of "cloverleaf shaped" zone of inhibition. The test organinsm was considered as Metallo-beta lactamase (MBL) positive. ${ }^{[14]}$

Imipenem(IMP)- EDTA Combined disc test: The test organisms are inoculated by lawn culture technique on the plates of Muller-Hinton agar(MHA) as recommended by CLSI. ${ }^{[13]} 10 \mu \mathrm{g}$ Imipenem Disk and 750 $\mu \mathrm{g}$ Imipenem-EDTA Disk(Hi-media SD281) are placed on the plate. The inhibition zones of the imipenem and imipenem-EDTA disks are compared after 16 to 18 hours of incubation at $37^{\circ} \mathrm{C}$. In the combined disc test, if the increase in inhibition zone with the imipenem and EDTA disc will be $\geq 7 \mathrm{~mm}$ than the imipenem disc alone, it is considered as MBL positive. ${ }^{[15]}$

MBL E test: The E-test MBL Strip contains a double sided seven-dilution range of IP(Imipenem) (4 to $256 \mu \mathrm{g} / \mathrm{ml}$ ) and Imipenem (1 to $64 \mu \mathrm{g} / \mathrm{ml}$ ) in combination with a fixed concentration of EDTA is considered as the most sensitive method for MBL detection]. The E-test was done according to manufacturer's instructions. MIC ratio of IP/ IPI(Imipenem+EDTA) of $>8$ or $>3 \quad \log$ dilutions indicates MBL production. ${ }^{[16]}$

\section{Results and Discussion}

Carbapenems have a broad spectrum of antibacterial activity. Hence, they are often used as a last resort in treatment. These are resistant to hydrolysis by most $\beta$-lactamases including extended spectrum $\beta$-lactamases (ESBL) and AmpC $\beta$ - lactamases. There has been an increase in reports of Carbapenem resistance in $P$. aeruginosa worldwide.

In India the first report of metallo beta lactamase was published from Bangalore, MS Ramaiah Medical College ${ }^{[17]} 12 \%$ of the isolates included in the study were resistant to both beta lactamase inhibitors and Carbapenem.100\% of these isolates were found to be metallo beta lactamase producer [17].

In India the studies done on metallo beta lactamase producing non fermenters are numerous. The prevalence of metallo beta lactamase producers among Carbapenem resistant isolates (Resistant to either or both Imipenem and Meropenem) in the present study was found to be $15 / 68(22.05 \%)$ The results vary all over the country. The rate of metallo beta lactamase production in our study is much lower compared to most of the other studies done in India. It has been reported as low as $7.5 \%^{[18]}$ to as high as $100 \%^{[17]}$.

The criteria for choosing the isolates for MBL screening are varied. Some studies have chosen Ceftazidime resistant strains for screening $\mathrm{MBL}^{[19,20,21]}$. Most of the studies have chosen Imipenem resistant strains for screening of MBL. In the present study strains resistant towards either or both Imipenem and Meropenem were included. 
Table.1 MBL screening test

\begin{tabular}{|c|c|}
\hline Organism & Negative \\
\hline Pseudomonas aeruginosa & $29 / 86(33.7 \%)$ \\
\hline
\end{tabular}

Table.2 Phenotypic confirmatory test

\begin{tabular}{|l|c|c|c|}
\hline \multicolumn{1}{|c|}{ Organisms } & $\begin{array}{c}\text { Modified hodge } \\
\text { test }\end{array}$ & $\begin{array}{c}\text { Imipenem(IMP)- } \\
\text { EDTA Combined disc } \\
\text { test: }\end{array}$ & E-test \\
\hline $\begin{array}{l}\text { Pseudomonas } \\
\text { aeruginosa }\end{array}$ & $15 / 21$ & $14 / 21$ & $19 / 21$ \\
\hline & & & \\
\hline
\end{tabular}

Fig.1 MBL positive by Modified hodge test

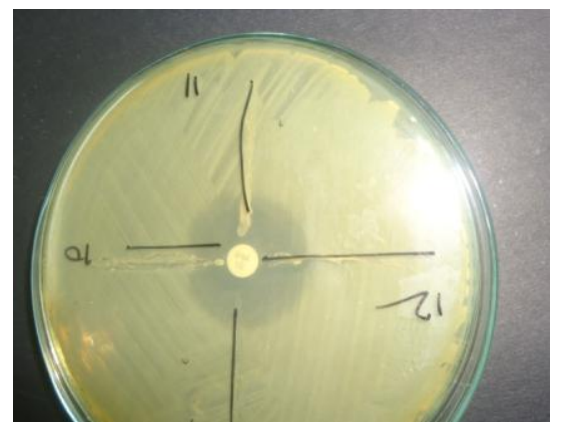

Fig.2 MBL positive by Imipenem (IMP)- EDTA Combined disc test

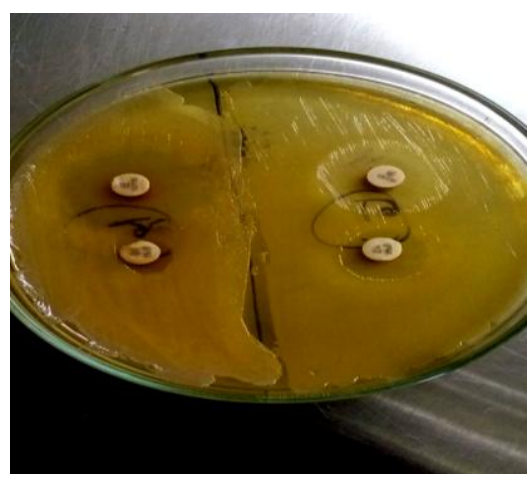

Fig.3 MBL positive by E-Test

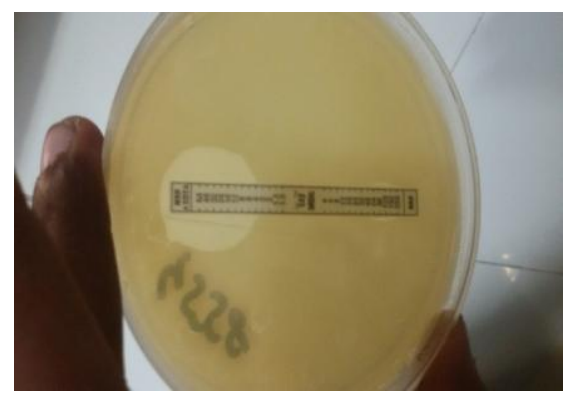


However all the isolates included in the study was resistant to Ceftazidime. 21/68(29.41\%) Pseudomonas aeruginosa showed screening test positive. The similar finding were seen by Buchende S etal (2012) and Renu S etal $(2010)^{[22,23]}$.

For screening of Metallo beta lactamase in the present study Double disc synergy test, Modified hodge test and Etest was done. [Figure-1 and figure-2]Modified hodge test(MHT) shows more positive MBL then that of Imipenem(IMP)- EDTA Combined disc test. Modified hodge test is recommended by CLSI for confirmation of MBL production. One study, shows that MHT had better sensitivity compared to $\mathrm{CDT}^{[24]}$. Where as Meropenem EDTA CDT to be a better test compared to $\mathrm{MHT}^{[22]}$.

In the present study E-test could detect MBL in the Imipenem resistant isolates correctly.[figure- 3] E-test showed high sensitivity as compared to Double disk synergy test and Modified hodge test. This is in accordance with the study done where all the isolates that screened positive for CDT where also positive by Etest ${ }^{[25]}$.

In conclusion, the study results demonstrate the serious therapeutic and epidemiological threat of the spread of metallo-beta-lactamase producing Pseudomonas aeruginosa. In this study, E-test was found to be best confirmatory tests followed by double disk synergy test and Modified Hodge test in this part of the country.

\section{References}

1. Ana Lucia, Peixoto de Freitas and Afonso Luis Barth. Antibiotic resistance and molecular typing of Pseudomonas aeruginosa: Focus on Imipenem. The Brazilian Journal of Infectious Diseases, 2002;6(1):1-7.
2. Bonomo RA, Szabo D. Mechanisms of multidrug resistance in Acinetobacter species and Pseudomonas aeruginosa. Clin Infect Dis, 2006;432: 49-56.

3. Bush K, Jacoby GA, Medeiros AA. A functional classification scheme for $b$ lactamases and its correlation with molecular structure. Antimicrobial Agents Chemother, 1995; 39:1211-33.

4. National Nosocomial Infections surveillance (NNIS) System Report, data summary from January 1992 through june 2004, issued October 2004. Am J Infect Control, 2004; 32:470-85.

5. Bell JM, Turnidge JD, Gales AC, Pfaller MA, Jones RN. Prevalence of extended spectrum beta-lactamase (ESBL) producing clinical isolates in the Asia-Pacific region and south Africa: regional results from SENTRY Antimicrobial surveillance program(1998-99). Diagn Microbiol Infect Dis, 2002; 42:193-8.

6. Falagas ME, Bliziotis IA. Pandrugresistant gram-negative bacteria: the dawn of the post-antibiotic era? Int $\mathbf{J}$ Antimicrob Agents, 2007; 29:630-6.

7. Nordmann P, Poirel L. Emerging carbapenemases in gram negative aerobes. Clin. Microbiol. Infect., 2002; 8: 321-333.

8. Walsh TR, Toleman MA, Poirel L, Nordman P. Metallo $\beta$-lactamases: the quiet before storm? Clin. Microbiol. Rev., 2005; 18: 306-325.

9. Deeba Bashir, Manzoor Ahmad Thokar,Bashir Ahmad Fomda, Gulnaz Bashir, Danish Zahoor, Shabir Ahmad and Abubaker S. Toboli. Detection of metallo-beta-lactamase

(MBL) producing Pseudomonas aeruginosa at a tertiary care hospital in Kashmir. African Journal of Microbiology Research, 2011; 5(2): 164-172. 
10. Valenza G, Joseph B, Elias J, Claus H, Oetterlein A, EngelHardt K, Turnwald D, Frosch M, Abele-Horn M Schoen C. First survey of Metallo- $\beta$-lactamases in clinical isolates of Pseudomonas aeruginosa in a German university hospital. Antimicrob Agents Chemother, 2010; 54: 3493-3497.

11. Lauretti L, M L Riccio, A. Mazzariol G. Cornaglia G. Amicosante, R.Fontana, G. M.Rossolini. Cloning and characterization of blaVIM, a new integron-borne metallo- $\beta$-lactamase gene from a Pseudomonas aeruginosa clinical isolate. Antimicrob. Agents chemother, 1999; 43:1584-90.

12. Nordmann P, Cuzon G, Naas T.The real threat of Klebsiella pneumoniae carbapenemase-producing

bacteria.Lancet Infect Dis., 2009; 9 :228-236.

13. CLSI. Performance Standards for Antimicrobial Susceptibility Testing;Twenty-Fourth Informational Supplement. CLSI document M100S24. Wayne, PA: Clinical and Laboratory Standards Institute; 2016.

14. Amjad A, Mirja IA, Abbasi SA, Farwa U, Malik N, Zia F. Modified Hodge Test: A simple and effective test for detection of carbepenemase production. Iran. J. Microbiol., 2011; 3(4): 189-193.

15. Yong D, Lee K, Yum JH, Shin HB, Rossolini GM, Chong Y. ImipenemEDTA disk method for differentiation of metallo- $\beta$-Lactamases producing clinical isolates of Pseudomonas spp. And Acinetobacter spp. J Clin Microbiol, 2002; 40:3798-801.

16. Walsh T, Bolmström A, Quarnström A et al. Evaluation of a new Etest for detecting metallo- $\beta$-lactamases in routine clinical testing. $J$ Clin Microbiol, 2002; 40:2755-3

17. Navaneeth BV, Sridaran D, Sahay D, Belwadi MR. A preliminary study on metallo-[beta]-lactamase producing Pseudomonas aeruginosa in hospitalized patients. Indian Journal of Medical Research, 2002; 116:264,

18. Gupta V, Datta P, Chander J. Prevalence of metallo- $\beta$ lactamase (MBL) producing Pseudomonas spp. and Acinetobacter spp. in a tertiary care hospital in India. Journal of Infection,2006; 52(5):311-4.

19. Hemalatha V, Sekar U, Kamat V. Detection of metallo betalactamase producing Pseudomonas aeruginosa in hospitalized patients. Indian Journal of Medical Research,2005; 122(2):148.

20. Arakawa Y, Shibata N, Shibayama K, Kurokawa H, Yagi T, Fujiwara H, Goto M. Convenient test for screening metallo- $\beta$-lactamase-producing gramnegative bacteria by using thiol compounds. Journal of Clinical Microbiology,2000;38(1):40-3.

21. Franklin C, Liolios L, Peleg AY. Phenotypic detection of carbapenemsusceptible metallo- $\beta$-lactamaseproducing gram-negative bacilli in the clinical laboratory. Journal of clinical microbiology, 2006; 44(9):3139-44.

22. Buchunde S, Mendiratta DK, Deotale V, Narang P. Comparison of disc and MIC reduction methods with polymerase chain reaction for the detection of metallo- $\beta$-lactamase in Pseudomonas aeruginosa. Indian journal of medical microbiology, 2012;30(2):170.

23. Renu G, Rajeev T, Smita S. The existence of metallo beta lactamases in carbapenem susceptible gram negative bacilli: A cause for concern. J Clin Diagn Res, 2010; 4: 2679-84.

24. Picão RC, Carrara-Marroni FE, Gales AC, Venâncio EJ, Xavier DE, Tognim MC, Pelayo JS. Metallo- $\beta$-lactamaseproduction in meropenem-susceptible Pseudomonas aeruginosa isolates: risk 
for silent spread. Memórias do Instituto Oswaldo Cruz.,2012;107(6):747-51.

25. Behera B, Mathur P, Das A, Kapil A, Sharma V. An evaluation of four different phenotypic techniques for detection of metallo- $\beta$-lactamase producing Pseudomonas aeruginosa. Indian journal of medical microbiology,2008;26(3):233.

\section{How to cite this article:}

Sujatha, R., Roma Goyal and Vikas Mishra. 2017. Detection of Metallo Beta Lactamase Producing Pseudomonas aeruginosa among Clinical Isolates. Int.J.Curr.Microbiol.App.Sci. 6(2): 1567-1573. doi: http://dx.doi.org/10.20546/ijcmas.2017.602.175 